\title{
KIMURA'S $Z$-TERM AND THE LIQUID CORE THEORY
}

\author{
YASUJIRO WAKO \\ International Latitude Observatory of Mizusawa, Iwate, Japan
}

\begin{abstract}
Kimura (1902) pointed out that there might be an unknown cause other than polar motion which would produce an apparent latitude variation, and introduced the $Z$-term as, $\Delta \phi=X \cos \lambda+$ $+Y \sin \lambda+Z$.

The theoretical results by Jeffreys-Vicente (1957) and Molodensky (1961) have shown that the effect of a liquid core of the Earth may increase the coefficients of the semi-annual solar nutation term $(2 \odot)$ which is involved in the diurnal nutation (the so-called Oppolzer term) by 0.02 . It is reasonable to accept this correction which will appear in the $Z$-term with an argument of $(2 \odot-\alpha)$.

From comparison of the observed amplitudes and the phase angles of the annual $Z$-terms derived from the ILS data, it is concluded that the argument of the principal annual term in $Z$ is $(2 \odot-\alpha)$ and not $\odot$. The following results were obtained for the annual $Z$-term for 1955-1966 from the analysis of data by two independent methods (Wako, 1970): 0!0137 $\sin (2 \odot-\alpha+2.2), 0$ ":0203 $\sin (2 \odot-\alpha+4.3)$.

Melchior (1970) proposed another effect of the Earth's liquid core for the annual nutation in obliquity, thus a term such as a $\sin (O+\alpha+A)$ would appear in the $Z$-term and it might cause a part of semi-annual $Z$-term. For the determination of these corrections, analysis of Kimura's $Z$-term is the most effective method.
\end{abstract}

1. Kimura (1902a, b) pointed out that there might be an unknown cause other than polar motion which would produce an apparent latitude variation, and introduced the $Z$-term as,

$$
\Delta \varphi=X \cos \lambda+Y \sin \lambda+Z .
$$

Kimura (1902a, b) reported that the $Z$-term in the latitude variation of the International Latitude Service (ILS) stations had an annual period, the amplitude of which was nearly equal to 0.03 , and that the value of $Z$ vanished around the equinoxes and attained its maximum and minimum around the solstices.

From comparison of the observed amplitudes and the phase angles of the annual $Z$-terms derived from the ILS data, it is concluded that the argument of the principal annual term in $Z$ is $(2 \odot-\alpha)$ and not $\odot$, where $\odot$ is the Sun's mean longitude and $\alpha$ the mean right ascension of the stars observed. The folllowing results were obtained for the annual $Z$-term for 1955-1966 from the analyses of data by two independent methods (Wako, 1970):

$$
0.0137 \sin (2 \odot-\alpha+2.2): 0.0203 \sin (2 \odot-\alpha+4.3) .
$$

It is to be noted that the phases are very close to zero.

Jeffreys and Vicente (1957a, b) and Molodensky (1961) studied the effects of liquid core on the rotation of the Earth. In these theories, they developed the motion of the axis of figure of the Earth with respect to space. The theoretical results by JeffreysVicente $e t$ al. have shown that the effect of a liquid core of the Earth may increase the coefficients of the semi-annual solar nutation term $(2 \odot)$ which is involved in the diurnal nutation (the so-called Oppolzer (1882) term) by 0.02 . It is reasonable to accept 
this correction which will appear in the $Z$-term with an argument of $(2 \odot-\alpha)$. The observed Kimura annual Z-term ((A) and (B)) is in good agreement with the theoretical results.

As the monthly mean latitudes are used in the calculation of the polar ccordinates, terms which include lunar longitude $\left(L_{\S}\right)$ in the argument must be neglected. The 19-yearly nutation term should be neglected since short interval data are used for the values of $Z$. For the cases of the 19-yearly and fortnightly nutations, comparison of the theoretical and observed values has been already made by Fedorov (1963), and is not discussed here.

The constant term in $Z$ is explained by the errors of the catalog used, when we compare Boss's General Catalog with the Melchior and Dejaiffe (1969) Catalog for the ILS stars (Wako, 1969a, b).

2. Melchior (1970) proposed another effect of the Earth's liquid core for the annual nutation in obliquity.

\begin{tabular}{lcc} 
& Annual Nutation Term: $(L-\Gamma)$ & \\
& Longitude $(\sin \varepsilon x)$ & Obliquity \\
Liquid core & $+0 ! 0579$ & -0.0056 \\
Rigid Earth & $+0 ! 0502$ & 0 \\
\hline Difference & $+0 ! 0077$ & $-0 ! 0056$
\end{tabular}

$L-\Gamma=$ Mean anomaly of the Sun (365.26 days)

$$
=\odot+78: 8 \text {. }
$$

Thus the errors in the values of the coefficients will be included in the $Z$-term as follows

$$
\begin{aligned}
& a \sin (L-\Gamma) \cos \alpha+b \cos (L-\Gamma) \sin \alpha= \\
& \quad=0.0011 \sin (\odot-\alpha+78.8)+0.0066 \sin (\odot+\alpha+78.8) .
\end{aligned}
$$

The $(\odot-\alpha)$ term is found together with the so-called closing sums, since the difference between the Sun's longitude and the right ascension is always constant for all the ILS stations. The $(\odot+\alpha)$ term has the semi-annual period and so it can be separated from the $Z$-term. However, Thomas (1964) pointed out that the diurnal free nutation has a nearly semi-annual period, that is 204 days. Then we must separate the semi-annual term and the diurnal free nutation term simultaneously. The results

\begin{tabular}{|c|c|c|c|c|}
\hline \multirow[t]{2}{*}{ Period } & \multicolumn{2}{|c|}{ Diurnal free nutation } & \multicolumn{2}{|c|}{ Semi-annual term } \\
\hline & Amplitude & Phase & Amplitude & Phase \\
\hline 1949-'54 & $\begin{array}{r}0.0078 \\
\pm 0.0026\end{array}$ & $\begin{array}{r}85.1 \\
\pm 20.7\end{array}$ & $\begin{array}{r}0.0061 \\
\pm 0.0026\end{array}$ & $\begin{array}{r}237.9 \\
\pm 24.2 \\
\end{array}$ \\
\hline 1955-'66 & $\begin{array}{r}0.0014 \\
\pm 0.0017\end{array}$ & $\begin{array}{r}82: 6 \\
\pm 69.3 \\
\end{array}$ & $\begin{array}{r}0.0104 \\
+0.0013\end{array}$ & $\begin{array}{r}345.9 \\
\pm \quad 8.5\end{array}$ \\
\hline
\end{tabular}
derived from the $Z$-term are given in Table $I$.

TABLE I 
Looking at the values of each period, we notice that they are not in good agreement. The possibility of determining the annual nutation and the diurnal free nutation from the $Z$-term exists, of course, and this would furnish another check on the liquid core theory.

However, it is likely that the determination from observation will present still more difficulties than the case of the semi-annual mutation term in the $Z$-term since the theoretical corrections for the annual nutation and the diurnal free nutation are small effects (Vicente and Jeffreys 1964).

3. The close relation between Kimura's annual $Z$-term and the theoretical semiannual solar nutation term based on the hypothesis of liquid core of the Earth is suggested. The hypothesis of a liquid core might be confirmed from the Kimura's Z-term of the long series of observations obtained by the ILS during the past seventy years in a uniform manner was commenced at the International Latitude Observatory of Mizusawa. The Melchior and Dejaiffe Catalog (1969) of the ILS stars may be very useful for these recalculations.

\section{References}

Fedorov, E. P.: 1963, in Nutation and Forced Motion of the Earth's Pole (Pergamon, New York, transl. by B. S. Jeffreys).

Jeffreys, H. and Vicente, R. O.: 1957a, Monthly Notices Roy. Astron. Soc. 117, 142.

Jeffreys, H. and Vicente, R. O.: 1957b, Monthly Notices Roy. Astron. Soc. 117, 162.

Kimura, H.: 1902a, Astron. J. 27, 107.

Kimura, H.: 1902b, Astron. Nachr. 158, 234.

Melchior, P.: 1970, 'Precession-Nutations and Tidal Potential', in IAU Colloquium No. 9 (System of Astronomical Constants), Heidelberg.

Melchior, P. and Dejaiffe, R.: 1969, Ann. Obs. Roy. Belgique, 3e Serie, 10, Fasc. 3.

Molodensky, M. S.: 1961, Comm. Obs. Roy. Belgique 188, S., G., 58, 25.

Oppolzer, T. von: 1882, Lehrbuch zur Bahnbestimmung 1, 126.

Thomas, D. V.: 1964, Nature 201, 481.

Vicente, R. O. and Jeffreys, H.: 1964, Nature 204, 120.

Wako, Y.: 1969a, Acad. Roy. Belg. Bull. Cl. Sci. Se Serie, 55, 357.

Wako, Y.: 1969b, Publ. Int. Lat. Obs. Mizusawa 7, No. 1, 51.

Wako, Y.: 1970, Publ. Astron. Soc. Japan 22, 525.

\section{DISCUSSION}

P. Melchior: Several authors have determined free diurnal nutation without consideration of correction of forced nutation due to liquid core effects. Is it your opinion that one cannot treat these effects separately?

Y. Wako: No. I think that separating these nutations requires the utmost care in the reduction of observations and the selection of the program stars. 